\title{
Influência do Exercício Leve na Pressão Arterial de Idosos em uso de Anti- Inflamatórios Não Hormonais
}

\section{Influence of Light Physical Exercise in Elderly Arterial Pressure and the use of non Hormonal Anti-Inflammatory Medicine}

\author{
Artigo \\ Original \\ Original \\ Paper \\ Walter Luiz M. S. da Fonseca ${ }^{1}$ \\ Marcilene M. A. Fonseca ${ }^{2}$ \\ Ivanete R. S. de Oliveira ${ }^{3}$ \\ Denise B. Miranda ${ }^{4}$ \\ Pedro S. do Prado Júnior ${ }^{4}$
}

Palavras-chaves:
Exercício Físico
Idoso
Drogas
Anti-inflamatórias

Resumo

Anti-inflamatórias

Os autores apresentam uma análise da variação da pressão arterial em indivíduos idosos hipertensos, submetidos a exercícios leves, praticados sob orientação de professores de educação física. Confirmou-se o registrado na literatura médica que a prática dos exercícios, em médio prazo auxilia na redução da pressão, entretanto, um grupo de pacientes em uso de antiinflamatórios não hormonais não apresentou os benefícios desejados, ou, talvez, até tenha tido o seu quadro hipertensivo agravado.

\begin{abstract}
The authors present an analysis about the variation in arterial pressure in hypertensive elderly individuals, under light physical exercises, practiced under Physical Education teachers' orientation. It was confirmed what is registered in medical literature, that is, the exercises practice, in middle time, helps in reducing the arterial pressure. However, a group of patients using non-hormonal anti-inflammatory medicines has not presented the desired benefits, or, maybe, they have had their hypertensive problem even worse.
\end{abstract}

Key words:

Physical Exercise

Elderly

Anti-inflammatory Drugs

\section{Introdução}

Considera-se exercício físico a atividade que é realizada com movimentos orientados e repetidos de forma sistemática, ocasionando aumento do consumo de oxigênio pela massa muscular. Como conseqüência do exercício físico há uma série de respostas fisiológicas, em particular, no sistema cardiovascular, mas também em outros sistemas orgânicos (respiratório, músculo articular entre outros).

Podemos classificar os efeitos fisiológicos do exercício em agudos e crônicos. Os efeitos agudos, por sua vez, podem ser produzidos em associação direta com o exercício, como a elevação da freqüência cardíaca e da ventilação pulmonar, com produção de calor e em conseqüência, sudorese. Os efeitos agudos podem apresentar manifestações nas 24 a 72 horas seguintes, com a discreta redução dos níveis tensionais, a expansão do volume plasmático e o aumento da sensibilidade insulínica na musculatura esquelética.

É, portanto, reconhecido que a atividade física de intensidade moderada ou vigorosa apresenta benefícios para a saúde.

\footnotetext{
"Nos indivíduos hipertensos, o exercício aeróbio regular pode exercer reduções modestas na pressão arterial, em média de $5 / 2 \mathrm{mmHg}$. Apesar de ser pequena em magnitude, estas reduções podem persistir por até 16 horas após um período de exercício aeróbio."
}

(Taylor-Tolbert N, et al.)

\footnotetext{
${ }^{1}$ Especialista - Professor Titular de Diagnóstico Clínico - Medicina - UniFOA

${ }^{2}$ Especialista - Professora Assistente de Diagnóstico Clínico - Medicina - UniFOA

${ }^{3}$ Mestre - Educação Física - UniFOA

${ }^{4}$ Acadêmicos - Medicina - UniFOA
} 
Os efeitos crônicos do exercício regular vão igualmente influenciar beneficamente o sistema cardiovascular, com aumento do débito cardíaco, fluxo sanguíneo redistribuído com conseqüente melhora da perfusão muscular. $\mathrm{O}$ aumento do débito cardíaco ocasiona aumento da pressão arterial sistólica enquanto a redistribuição do fluxo sanguíneo ocasiona diminuição da pressão arterial diastólica, com conseqüente diminuição da resistência periférica.

Inicialmente, a nossa intenção era estudar o comportamento da pressão arterial em pessoas idosas, hipertensas, submetidas a exercícios regulares e orientadas, para verificar qual é o predomínio: a elevação da pressão pelo aumento do débito cardíaco ou a diminuição da pressão arterial pela diminuição da resistência periférica. Para tanto, foi considerada a pressão arterial média, conforme descrito em Material e Métodos.

Entretanto, o estudo dos casos demonstrou que um grupo de indivíduos (Grupo 3) não apresentou as melhoras pressóricas desejadas, ou piorou. Este grupo estava em uso de antiinflamatórios não hormonais (AINH).

\section{Materiais e Métodos}

Cerca de 100 indivíduos inscritos no programa do UniFoa destinado a idosos, portadores de hipertensão arterial, foram convidados a participar do estudo, que tinha como objetivo inicial validar o apresentado pela literatura médica e de educação física de que o exercício físico leve, orientado, melhora a pressão.

Destes, 28 aceitaram participar, assinaram o termo de concordância e participaram das várias avaliações periódicas. Todos estavam inscritos nas modalidades natação e caminhada em superfície plana.

$\mathrm{O}$ protocolo incluiu avaliação clínica prévia, constando de exame clínico (anamnese orientada e exame físico geral) seguido de rotina laboratorial (hemograma, dosagem do colesterol, creatinina e glicose). Os indivíduos selecionados eram hipertensos já diagnosticados e estavam previamente medicados para hipertensão arterial. Eles foram inicialmente divididos em dois grupos:
Grupo 1 - hipertensos com a pressão bem controlada pelos medicamentos.

Grupo 2 - hipertensos com a pressão mal controlada pelos medicamentos. Este grupo recebia a medicação regularmente, alegava fazer o uso correto mas a pressão não se mantinha em níveis ideais, sendo atribuído ao sedentarismo.

As medidas da Pressão Arterial consideradas no estudo foram as obtidas na:

- Data de inscrição no programa pelo idoso. A pressão foi aferida pelo médico do programa no ambulatório do Campus Universitário.

- Data da conclusão do estudo (intervalo de 3 a 24 meses). A pressão foi aferida no ambulatório do Curso de Educação Física do Campus Universitário, após a recuperação do exercício (banho e mudança de roupa no caso da natação).

- Foi utilizada a pressão arterial média, obtida pela fórmula (PA sistólica $+2 \mathrm{x}$ PA diastólica dividido por 3), medida no antebraço pelos co-autores acadêmicos de medicina, previamente treinados e obedecendo ao padrão.

A Pressão Arterial foi aferida de acordo com os critérios propostos pela Sociedade Brasileira de Cardiologia, sendo utilizados dois esfigmomanômetros Tycos $®$ calibrados antes das aferições.

A distribuição por idade e por sexo não apresentou interesse para a pesquisa: a idade variou entre 50 e 60 anos, e a distribuição por sexo foi homogênea.

A análise dos resultados fez com que se dividisse o Grupo 2 em dois, ficando no grupo 2 os indivíduos hipertensos que obtiveram melhora com o exercício e o Grupo 3 com o que não obtiveram melhora.

\section{Resultados}

A análise de entrada dos indivíduos e do segmento permitiu a divisão em três grupos:

Grupo 1: 13 Indivíduos hipertensos bem controlados antes dos exercícios, em que não houve variação da PA com a atividade física. 
Grupo 2: 11 Indivíduos com Pressão Arterial moderadamente elevada no primeiro exame, apesar do uso de medicamento, tornando-se normal após a prática de exercício físico.

Grupo 3: 4 indivíduos - A Pressão Arterial era normal ou normal-alta no primeiro exame, tendo se elevado após a prática de exercício físico.

Os resultados estão contidos nos quadros 1 a 9

Quadro 1-Grupo 1-medida da pressão arterial média antes e depois em cada indivíduo

\begin{tabular}{|l|c|c|}
\hline & Antes & Depois \\
\hline Idoso 1 & 96,7 & 85,7 \\
\hline Idoso 2 & 100 & 93,3 \\
\hline Idoso 3 & 96,7 & 94 \\
\hline Idoso 4 & 86,7 & 86,7 \\
\hline Idoso 5 & 85 & 99,3 \\
\hline Idoso 6 & 101,7 & 101,7 \\
\hline Idoso 7 & 106,7 & 106,7 \\
\hline Idoso 8 & 93,3 & 83,3 \\
\hline Idoso 9 & 93,3 & 100 \\
\hline Idoso 10 & 100 & 96,6 \\
\hline Idoso 11 & 103,3 & 93,3 \\
\hline Idoso 12 & 100 & 93,3 \\
\hline Idoso 13 & 100 & 88,7 \\
\hline
\end{tabular}

Quadro 2-Grupo 1-média da pressão arterial média antes e depois

\begin{tabular}{|l|c|c|}
\hline & Média & DP \\
\hline Antes & 97,18462 & 6,252179 \\
\hline Depois & 94,04615 & 6,816844 \\
\hline
\end{tabular}

Quadro 3-Grupo 1 - gráfico comparativo da pressão arterial média antes e depois

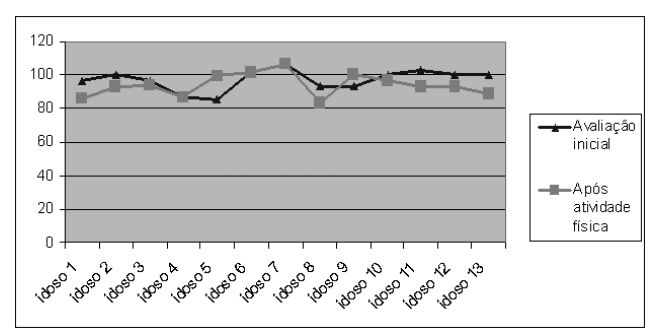

Quadro 4-Grupo 2-medida da pressão arterial média antes e depois em cada indivíduo

\begin{tabular}{|l|c|c|}
\hline & Antes & Depois \\
\hline Idoso 14 & 116,7 & 83,3 \\
\hline Idoso 15 & 123,3 & 86,7 \\
\hline Idoso 16 & 121,7 & 86,7 \\
\hline Idoso 17 & 140 & 113,3 \\
\hline Idoso 18 & 110 & 100 \\
\hline Idoso 19 & 106,7 & 88 \\
\hline Idoso 20 & 130 & 116,7 \\
\hline Idoso 21 & 120 & 103,3 \\
\hline Idoso 22 & 113,3 & 106 \\
\hline Idoso 23 & 123,3 & 110 \\
\hline Idoso 24 & 120 & 90 \\
\hline
\end{tabular}

Quadro 5-Grupo 2-média da pressão arterial média antes e depois

\begin{tabular}{|l|c|c|}
\hline & Média & DP \\
\hline Antes & 120,4545 & 9,246985 \\
\hline Depois & 96,90909 & 11,6053 \\
\hline
\end{tabular}

Quadro 6-Grupo 2- gráfico comparativo da pressão arterial média antes e depois

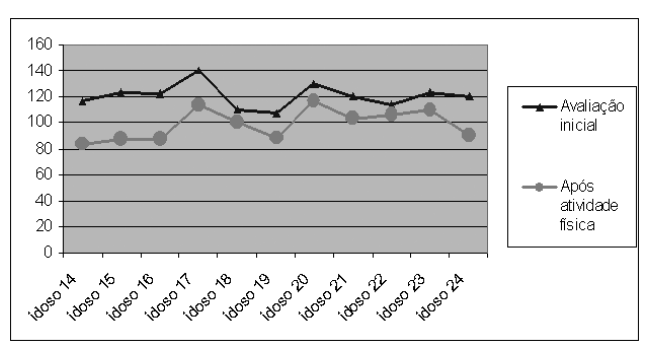

Quadro 7-Grupo 3-medida da pressão arterial média antes e depois em cada indivíduo

\begin{tabular}{|l|c|c|}
\hline & Antes & Depois \\
\hline Idoso 25 & 100 & 111,3 \\
\hline Idoso 26 & 116,7 & 159,3 \\
\hline Idoso 27 & 93,3 & 120 \\
\hline Idoso 28 & 106,7 & 120 \\
\hline
\end{tabular}

Quadro 8-Grupo 3-média da pressão arterial média antes e depois

\begin{tabular}{|l|c|c|}
\hline & Média & DP \\
\hline Antes & 104,175 & 9,982443 \\
\hline Depois & 127,65 & 21,49488 \\
\hline
\end{tabular}

Quadro 9 - Grupo 3 - gráfico comparativo da pressão arterial média antes e depois

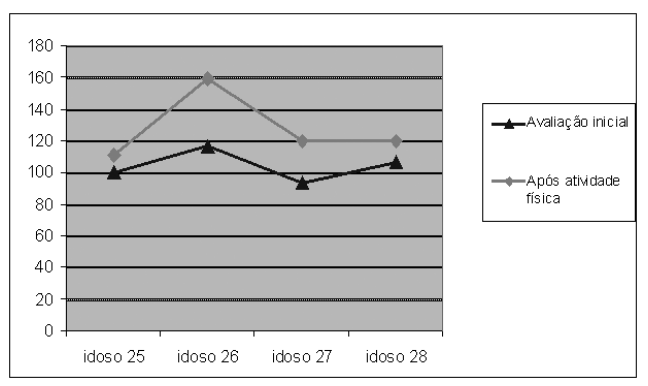




\section{Discussão}

A análise do primeiro e do segundo grupo atendeu o que se esperava do exercício físico - redução da pressão arterial média. A análise do terceiro grupo apresentou um resultado que surpreendeu, pois os pacientes tiveram elevação da pressão arterial média.

Como os grupos eram uniformes do ponto de vista clínico, partiu-se para a pesquisa de um fator que pudesse explicar a não conformidade do resultado. O estudo dos prontuários demonstrou que todos os do terceiro grupo estavam em uso de antiinflamatórios não hormonais devido a dores articulares.

Como os AINH são apontados na literatura médica como substâncias capazes de interferir no controle da pressão arterial, os autores consideraram este fator como a provável causa da não conformidade.

\section{Conclusão}

A prática regular de exercícios físicos demonstrou ser um fator de melhora dos níveis de pressão arterial, que deve ser coadjuvante dos tratamentos farmacológico edietético, porém, ouso concomitante de antiinflamatórios não hormonais pode contribuir para neutralizar os benefícios, ou mesmo agravar o quadro pressórico.

Esta constatação nos conduz a recomendação de se introduzir no protocolo de exame médico de idosos candidatos a exercício físico o interrogatório sobre o uso de AINH. Essa recomendação reveste-se de maior importância nos idosos sedentários, portadores de dores articulares ou musculares, inclusive devido ao hábito da auto-medicação, costume sabidamente popular na nossa população.

Certamente, um estudo mais apurado dessa interferência deve ser feito para validar a hipótese, uma vez que a própria dor representa um fator adicional de stress e pode limitar a prática correta do exercício. Outros fatores farmacológicos, as interações medicamentosas ou a retenção salina induzida pelos AINH merecem ser pesquisados.
CIOlAC, EG; GUIMARÃES, GV - Physical exercise and metabolic syndrome. Rev Bras Med Esporte, July/Aug. 2004, vol.10, no.4, p.319-324. ISSN 1517-8692.

IZDEBSKA, E;I CYBULSKA, I; IZDEBSKIR, J; MAKOWIECKA-CIESLA M; TRZEBSKI, A - Effects of moderate physical training on blood pressure variability and hemodynamic pattern in mildly hypertensive subjects. Physiol Pharmacol, December 1, 2004; 55(4): 713-24.

LIMA, EG; HERKENHOFF, F; VASQUEZ, EC - Ambulatory blood pressure monitoring in individuals with exaggerated blood pressure response to exercise, influence of exercise training. Arq. Bras. Cardiol., Apr. 1998, vol.70, no.4, p.243-249. ISSN 0066-782X.

MONTEIRO, MF; SOBRAL FILHO, DC Physical exercise and blood pressure control. Rev Bras Med Esporte, Nov./Dec. 2004, vol.10, no.6, p.513-516. ISSN 1517-8692.

PINTO, VLM; MEIRELLES, LR; FARINATTI, PTV - Influence of domestic and community exercise programs on the physical fitness, arterial blood pressure, and biochemical variables in hypertensive patients. Rev Bras Med Esporte, Sept./Oct. 2003, vol.9, no.5, p.267274. ISSN 1517-8692.

SANTOS, BMR; GONÇALVES, RM; SILVA, $A R$ et al. Influence of physical exercise and sodium intake on arterial pressure and cardiac hypertrophy in rats. Rev. Hosp. Clin., Aug. 1999, vol.54, no.4, p.111-114. ISSN 0041-8781.

STEWART, KJ; BACHER, AC; TURNER, KL; JLFLEG,HEES, PS; SHAPIRO,EP;TAYBACK, M; OUYANG, P - Effect of exercise on blood pressure in older persons: a randomized controlled trial. Archives of Internal Medicine, April 11, 2005; 165(7): 756-62.

TAYLOR-TOLBERT N, DENGEL D, BROWN $M$, et al: Ambulatory blood pressure after acute exercise in older men whith essencial hypertension. Am J Hypertens 2000;13:44-51.

Informações bibliográficas:

Conforme a NBR 6023:2002 da Associação Brasileira de Normas Técnicas (ABNT), este texto científico publicado em periódico eletrônico deve ser citado da seguinte forma:

FONSECA, Walter Luiz M. S. da ; FONSECA, Marcilene M. A. ; OLIVEIRA, Ivanete R. S. de ; MIRANDA, Denise B.; PRADO JUNIOR, Pedro S. do. Influência do Exercício Leve na Pressão Arterial de Idosos em uso de Anti-Inflamatórios Não Hormonais. Cadernos UniFOA, Volta Redonda, ano 2, $\mathrm{n}^{\circ}$. 5, dez. 2007. Disponível em: <http://www.unifoa.edu.br/pesquisa/caderno/edicao/05/84.pdf> 